\section{Conhecimentos sobre o uso de contraceptivos e prevenção de DST: a percepção de mulheres imigrantes}

\author{
Knowledge of contraceptive methods and STD \\ prevention among immigrant women
}

Abstract

Sexual and reproductive health is a major public health concern, since it involves individual health and wellbeing and affects the socioeconomic level of societies. Socially underprivileged groups like immigrants may be more exposed to sexual and reproductive health problems. The current study examines key aspects of sexual and reproductive health among Brazilian and African immigrant women in Portugal, particularly contraceptive methods, sexually transmitted diseases, and condom use. Data were collected through focus groups with childbearing-age immigrant women living in Portugal and originally from Brazil or Portuguese-speaking African countries. The results highlight the participants' perceptions and knowledge concerning the respective issues and identify aspects to be considered in order to better meet their sexual and reproductive health needs.

Sexual and Reproductive Health; Women's Health; Emigrants and Immigrants; Communicable Disease Prevention
Cristianne Maria Famer Rocha 1,2 Sonia Ferreira Dias 3 Ana Filipa Gama ${ }^{3}$

\section{Introdução}

No quadro atual da globalização, os movimentos migratórios têm ganhado uma crescente importância. O reconhecimento de que é necessário compreender o fenômeno da distribuição e movimentação da população entre regiões, bem como a sua complexidade e o seu impacto, quer para os migrantes, quer para os países de acolhimento, levou o tema a se transformar em objeto de estudo na área da Saúde Pública e das Ciências Sociais e Humanas.

Não representando, por si mesma, um fator de risco para a saúde, a migração e as condições em que esta se processa podem aumentar a vulnerabilidade dos migrantes às doenças. Além disso, de acordo com o relatório A Passage to Hope: Women and International Migration 1 , o número de mulheres migrantes tem aumentado significativamente, o que coloca importantes desafios para a área da saúde, uma vez que, freqüentemente, as migrações de mulheres representam maiores riscos e vulnerabilidades com impacto para a saúde.

Dados de organizações internacionais têm realçado que o quadro de pobreza, de exclusão social, de populações vulneráveis e de falta de oportunidades aumenta os riscos e as susceptibilidades, contribuindo de forma determinante para o estado deficitário da saúde dos indivíduos 1,2. As questões de gênero, associadas a fatores como 
o tipo de migração, as condições a que o migrante é sujeito nos países de acolhimento, as políticas e atitudes destes países em relação à equidade $\mathrm{e}$ igualdade de oportunidades, o tipo de contato mantido com o país de origem e a possibilidade de retorno e reintegração, determinam o impacto da migração nas populações 3,4 .

A saúde sexual e reprodutiva representa também uma das principais preocupações da área da Saúde. Potenciais problemas nesta área afetam, direta ou indiretamente, a saúde e o bem-estar de indivíduos e de comunidades, além de representarem as principais causas de enfermidades e de morte das mulheres.

De complexa definição, a saúde sexual e reprodutiva engloba vários temas, como sexualidade, reprodução, direitos humanos e bem-estar, e exige uma reflexão sobre a forma como estes temas se inter-relacionam. Portanto, a saúde sexual e reprodutiva é um conceito holístico que inclui, para além da reprodução, as questões da saúde sexual e das relações pessoais 5,6. Integra aspectos relacionados com a sexualidade, de forma que esta seja positivamente enriquecedora e promova não só a comunicação, como também a relação entre os indivíduos 5,6,7,8,9.

Segundo a Organização Mundial da Saúde, a sexualidade é considerada um aspecto importante da vida humana e abrange áreas como o sexo, as identidades e papéis de gênero, a orientação sexual, o erotismo, o prazer, a intimidade e a reprodução ${ }^{8}$. É vivida e se expressa através de pensamentos, desejos, crenças, atitudes, valores, comportamentos, práticas e relações, podendo ser influenciada pela interação de fatores biológicos, emocionais, intelectuais, sócio-econômicos, culturais, históricos, religiosos, éticos, legais e políticos 8 . Sendo assim, a definição de saúde sexual e reprodutiva pressupõe a possibilidade/ necessidade de as pessoas terem uma vida sexual segura e com prazer, com capacidade de reprodução e liberdade de decisão.

Esta pressuposição está diretamente relacionada às questões dos direitos humanos, reconhecendo-se que todos os casais e indivíduos têm o direito à decisão livre e responsável nos aspectos relacionados com a reprodução (número de filhos, local e momento), sem discriminação, coerção ou violência, bem como o direito à informação e meios para tal 5,6. Nesta última condição, está implícito o direito das mulheres e dos homens à informação e ao acesso a métodos de planejamento familiar de sua escolha, que sejam seguros, eficazes e aceitáveis; tal direito se estende a serviços de saúde adequados, que permitam às mulheres ter gravidez e parto em segurança $\mathrm{e}$ ofereçam aos casais as melhores oportunidades de ter crianças saudáveis.
Outro aspecto a ser considerado em relação à saúde sexual e reprodutiva é a enorme disparidade existente sobre esta temática em cada país, entre países e entre gêneros 10 . Neste caso, a diferença dos papéis sociais de gênero (e suas variáveis sócio-econômicas e culturais) reflete-se muitas vezes em uma desigual exposição das mulheres a riscos para a saúde, quando comparadas com os homens 11. Segundo o Banco Mundial 12, as desigualdades de gênero comprometem a eficácia das políticas de desenvolvimento e de saúde; logo, a promoção da igualdade de gênero pode aumentar as oportunidades de desenvolvimento humano e eliminar sérios obstáculos à consecução dos Objetivos de Desenvolvimento do Milênio 10.

Por sua vez, o crescente aumento da imigração feminina tem acentuado a preocupação em relação às questões de saúde sexual e reprodutiva nesta população. Dados de organizações internacionais e de estudos epidemiológicos têm apontado para uma maior vulnerabilidade em saúde sexual e reprodutiva nas mulheres imigrantes 13,14. Investigações na área da saúde reprodutiva apontam para a existência de piores indicadores de saúde associados à população imigrante, concretamente no que se refere à mortalidade perinatal, baixo peso ao nascer e menor utilização de métodos contraceptivos 15,16,17. Mais especificamente, tem-se verificado que a reduzida e inadequada utilização desses métodos está freqüentemente associada à ocorrência de gravidez não desejada e interrupção voluntária desta 18,19 .

A compreensão de que muitos dos problemas da sexualidade e reprodução são particularmente acentuados nos grupos socialmente mais desfavorecidos, como são freqüentemente os imigrantes, colocou as questões da saúde sexual e reprodutiva no contexto do desenvolvimento de políticas de inclusão social e de luta contra a pobreza. Assim, é atualmente reconhecido que investir na saúde sexual e reprodutiva é vital para a diminuição da pobreza e melhoria das condições de vida dos indivíduos, em particular, dos imigrantes e suas famílias.

O desenvolvimento de programas efetivos em saúde requer que os comportamentos sexuais e reprodutivos sejam contextualizados na realidade em que a comunidade está inserida, determinada pela situação epidemiológica, mobilidade e dinâmicas sócio-culturais e econômicas criadas pelos fatores demográficos (migração e etnicidade) ${ }^{20}$. Ao mesmo tempo, são escassos os estudos sobre os conhecimentos, crenças, atitudes e comportamentos na área da sexualidade e da reprodução realizados em comunidades imigrantes, tornando difícil retirar conclusões acerca das diferenças culturais e étnicas que poderão existir 
nos determinantes dos comportamentos de risco. Diante disso, o planejamento de intervenções eficientes também encontra dificuldades, pois o desconhecimento da cultura e dos sistemas de crenças nas minorias étnicas, em particular alguns mitos, tabus e práticas culturais que envolvem os comportamentos sexuais, prejudicam a abordagem da questão da sexualidade junto às populações migrantes 21 .

Com base em tais premissas, o presente estudo teve como finalidade aprofundar o conhecimento sobre algumas questões que envolvem a saúde sexual e reprodutiva, especialmente o uso de contraceptivos e a prevenção às doenças sexualmente transmissíveis (DST) pelas imigrantes brasileiras e africanas residentes em Portugal. Objetivou, ainda, descrever os fatores de ordem cultural que afetam a saúde sexual e reprodutiva destas comunidades.

Em Portugal, os dados oficiais indicam que cerca de $45 \%$ da população imigrante é do sexo feminino e os grupos com maior representatividade são naturais do Brasil e de Países Africanos de Língua Oficial Portuguesa (PALOP) ${ }^{22}$. Segundo dados do Instituto Nacional de Estatística 23 , as mulheres imigrantes brasileiras residentes no país tendem a ter um nível educacional superior ao das mulheres africanas. Além disso, as brasileiras em Portugal desempenham predominantemente atividades domésticas, de restauração e de atendimento ao público, enquanto as imigrantes africanas tendem a desempenhar profissões pouco qualificadas, principalmente na área dos serviços gerais 23,24,25.

Para a concretização dos objetivos propostos, desenvolveu-se um estudo de cunho qualitativo, mediante a realização de grupos focais com mulheres imigrantes oriundas do Brasil e de PALOP, as quais, no decorrer deste texto, passarão a ser referidas como "mulheres brasileiras" e "mulheres africanas" ou simplesmente "brasileiras" e “africanas", respectivamente.

\section{Material e métodos}

Trata-se de pesquisa de nível exploratório e descritivo 26. Para a coleta dos dados, foi utilizada a técnica dos grupos focais (focus groups) ou grupos de discussão centrados em um tema. Grupo focal é uma técnica de investigação que permite a obtenção de informações de natureza qualitativa, a partir da realização de sessões de discussão com grupos de participantes que possuam características comuns. Permite, também, a identificação e o levantamento de opiniões que refletem o grupo em um período de tempo relativamente curto.
Os grupos focais têm sido utilizados no âmbito das ciências sociais e de saúde e, em particular, nas questões relacionadas com a investigação na área da saúde sexual e reprodutiva 27,28,29,30,31. A opção por essa técnica de coleta de dados está relacionada à intenção de melhor compreender as crenças, atitudes, estilos de vida e comportamentos de risco e proteção relacionados com a saúde sexual e reprodutiva, construídos em um contexto de elementos sociais, econômicos e culturais que caracterizam a realidade específica em que os indivíduos estão inseridos.

Com duração média de uma hora e trinta minutos, cada sessão foi registrada em áudio, após consentimento das participantes, de forma a maximizar a coleta de dados e permitir a transcrição integral das discussões realizadas, para posterior análise.

No início das sessões, em observância aos princípios éticos da Declaração de Helsinki e à legislação portuguesa, todas as participantes foram devidamente informadas sobre os objetivos gerais da investigação, o procedimento para coleta de dados e a forma como seria conduzida a reunião. Foi claramente explicitada a liberdade da participante para recusar participar ou desistir a qualquer momento da reunião, tendo sido obtido seu consentimento voluntário com garantia do anonimato e da confidencialidade dos dados.

No final de cada sessão, foram registradas as observações consideradas relevantes acerca da dinâmica e das interações dos participantes. Posteriormente, procedeu-se à transcrição integral das discussões dos grupos, de acordo com regras definidas anteriormente, garantindo a uniformização das transcrições.

Foi utilizada uma amostra intencional constituída por 35 imigrantes brasileiras e africanas, com a realização de seis grupos focais, três com imigrantes africanas e três com imigrantes brasileiras. A escolha das participantes nos grupos tomou em consideração os critérios de inclusão da amostra: mulheres imigrantes (provenientes do Brasil e de PALOP, cujos pais não tivessem nascido em Portugal), com idades compreendidas entre 18 e 45 anos, residentes em Portugal há mais de dois anos e com disponibilidade e vontade de participar da investigação. As participantes foram indicadas por organizações não governamentais, associações, instituições particulares de solidariedade social e centros sociais que desenvolvem atividades junto a imigrantes.

Das 35 mulheres participantes no estudo, 15 são brasileiras e 20 são africanas (destas, nove provêm de Cabo Verde, seis de Angola, quatro de São Tomé e Príncipe e uma da Guiné). A maioria das participantes brasileiras tem entre 21 e 29 
anos, ao passo que a maioria das participantes africanas tem entre 40 e 45 anos. As participantes brasileiras reportaram um nível educacional superior ao das participantes africanas. Quanto ao estatuto legal, cinco brasileiras e quatro africanas informaram que se encontravam, no momento da coleta de dados, em situação irregular ou em processo de regularização. Todas as mulheres brasileiras residem em Portugal há menos de dez anos, enquanto a maior parte das participantes africanas reside no país há mais de 15 anos (Tabela 1).

A análise dos resultados seguiu o procedimento da análise de conteúdo utilizada na metodologia qualitativa 32 . As intervenções realizadas pelas participantes, durante cada sessão, seguiram um roteiro preestabelecido que contemplava três dimensões e alguns subtemas (Tabela 2).

À medida que se prosseguia com a sistematização e decodificação dos dados, foram agrupadas as opiniões sobre diferentes temas, tendo em conta os consensos gerados nos grupos e as contribuições individuais. As diferentes intervenções constituíram categorias de análise, de acordo com a regularidade com que determinados elementos apareceram e se repetiram nas discussões. Na primeira fase, foram analisadas as discussões de cada grupo focal separadamente; depois foram analisadas todas as discussões, comparando-se os grupos de mulheres africanas com os de brasileiras.

\section{Resultados}

Os resultados deste estudo refletem os conhecimentos, as percepções, atitudes e práticas de imigrantes africanas e brasileiras em relação a alguns aspectos da saúde sexual e reprodutiva, e são organizados conforme duas categorias de análise: Uso de Métodos Contraceptivos e Prevenção de DST. Extratos dos discursos enunciados pelas participantes são apresentados nas duas categorias indicadas e, em seguida, discutidos, de forma a ilustrar os significados atribuídos por elas em relação a tais temáticas. São ainda destacadas as convergências e divergências existentes nos discursos dos dois grupos de participantes (mulheres imigrantes africanas e brasileiras).

Tabela 1

Características sócio-demográficas das participantes.

\begin{tabular}{|c|c|c|c|}
\hline Características & Africanas & Brasileiras & Total \\
\hline Total & 20 & 15 & 35 \\
\hline \multicolumn{4}{|l|}{ Idade (anos) } \\
\hline $21-29$ & 2 & 8 & 10 \\
\hline $30-39$ & 8 & 4 & 12 \\
\hline $40-45$ & 10 & 3 & 13 \\
\hline \multicolumn{4}{|l|}{ Nível educacional } \\
\hline 1/2o ciclos do Ensino Básico & 16 & - & 16 \\
\hline 3o ciclo do Ensino Básico & 3 & - & 3 \\
\hline Ensino Secundário & 1 & 12 & 13 \\
\hline Ensino Superior & - & 3 & 3 \\
\hline \multicolumn{4}{|l|}{ Estatuto legal } \\
\hline Nacionalidade portuguesa & 3 & 1 & 4 \\
\hline Situação regular & 13 & 9 & 22 \\
\hline Situação irregular & 4 & 5 & 9 \\
\hline \multicolumn{4}{|c|}{ Tempo de permanência em Portugal (anos) } \\
\hline $2-5$ & 1 & 11 & 12 \\
\hline $6-10$ & 7 & 4 & 11 \\
\hline $11-15$ & 3 & - & 3 \\
\hline$\geq 16$ & 9 & - & 9 \\
\hline \multicolumn{4}{|l|}{ Situação profissional } \\
\hline Empregado & 18 & 15 & 33 \\
\hline Desempregado & 2 & - & 2 \\
\hline
\end{tabular}


Tabela 2

Dimensões e subtemas dos grupos focais.

\begin{tabular}{|c|c|}
\hline Dimensões & Subtemas \\
\hline \multirow[t]{9}{*}{ Temáticas de saúde sexual e reprodutiva } & Conhecimento sobre planejamento familiar \\
\hline & Uso de métodos contraceptivos \\
\hline & Infecções sexualmente transmissíveis e uso do preservativo \\
\hline & Gravidez e maternidade \\
\hline & Interrupção voluntária da gravidez \\
\hline & Informação em saúde sexual e reprodutiva \\
\hline & Estado de saúde geral \\
\hline & Relações conjugais \\
\hline & Experiências de violência \\
\hline \multirow[t]{2}{*}{ Processo de migração } & Experiência de migração \\
\hline & Dificuldades no país de acolhimento \\
\hline \multirow[t]{5}{*}{ Acesso e utilização dos serviços de saúde } & Padrões de utilização dos serviços de saúde \\
\hline & Comparação entre a prestação dos cuidados de saúde no país de origem e no de \\
\hline & acolhimento (Portugal) \\
\hline & Barreiras no acesso e utilização dos serviços de saúde \\
\hline & Facilidades no acesso e utilização dos serviços de saúde \\
\hline
\end{tabular}

\section{Uso de métodos contraceptivos}

Em primeiro lugar, é preciso destacar que os resultados obtidos com esta investigação refletem o nível educacional das participantes, ou seja, quanto mais escolarizadas são (caso das brasileiras), mais conhecem sobre esta temática.

Nos grupos focais realizados, as brasileiras, de uma forma geral, reportam conhecer os diferentes métodos contraceptivos, reconhecem a sua eficácia, apresentam atitudes favoráveis à sua utilização e afirmam que iniciaram seu uso no Brasil (antes de terem migrado). Acrescentam que continuam usando, em Portugal, os contraceptivos indicados, seguindo a orientação que lá obtiveram: "eu trouxe do Brasil, cheguei na farmácia e disseram que só era receitado lá" (participante brasileira). "A orientação que eu tenho era a que eu trazia do Brasil” (participante brasileira).

Por outro lado, confirmando o que alguns estudos indicam em relação à persistente falta de conhecimento sobre os métodos contraceptivos disponíveis e eficazes, entre as populações imigrantes 33,34 , um grupo considerável de africanas revela a existência de lacunas no conhecimento sobre os métodos contraceptivos: "a pílula, além de prevenir a gravidez, previne alguma doença?” (participante africana). "Eu nunca tomei pílula; $e$ se eu tomar e engravidar?” (participante africana).

Ao mesmo tempo, as participantes africanas referiram, durante a realização dos grupos focais, uma série de crenças associadas à ineficácia dos métodos contraceptivos e à percepção de que são prejudiciais à saúde:

\section{- Crenças associadas à ineficácia dos métodos:}

"Eu usava pílula, e apanhava sempre gravidez" (participante africana). "Quando eu cheguei aqui (...), voltei a meter o outro aparelho (...); não chegou nem seis meses e eu tinha a minha filha. Engravidei com aparelho. Diziam que o aparelho português é que era bom, e eu engravidei com o aparelho português" (participante africana).

\section{- Crenças de que o uso de métodos contraceptivos é prejudicial à saúde:}

“Às vezes, há pessoas que não se dão com a pílula e começam a entupir, a entupir, e depois correm o grande risco de não terem mais filhos" (participante africana). "A minha prima pôs o aparelho (...) para não ter mais filhos [e] agora tem problemas graves, (...) tem medo de morrer porque ela corre risco de vida (...)" (participante africana). "É pior porque aquilo começa a apodrecer e a criar infecções" (participante africana).

Nestes grupos, é possível perceber também que as crenças associadas aos métodos contraceptivos contribuem de forma determinante para a sua não-utilização: "Eu quero minha saúde livre, livre de comprimido, livre de tudo (...)” (par- 
ticipante africana). "Os comprimidos por dentro começam a fazer uma camada do nosso organismo, e depois correm o grande risco de não poderem ter mais filhos (...). Por isso (...), nunca tomei, não quero tomar" (participante africana).

Um estudo de De Vries (1999, apud Janssens et al. 9) revelou que, por vezes, as mulheres de alguns grupos étnicos interrompem o uso de contracepção oral por acreditarem que o medicamento pode provocar câncer. Estas evidências são importantes, pois a falta de conhecimento pode determinar, direta ou indiretamente, uma fraca ou incorreta utilização dos métodos contraceptivos 35,36 .

\section{Prevenção às DST}

Em relação às DST, os resultados do estudo também apontam para a existência de lacunas importantes no conhecimento sobre a transmissão e prevenção dessas doenças, sobretudo nas participantes africanas. Tal falha produz implicações na adoção de práticas sexuais saudáveis ou de estratégias preventivas: “Ah! é uma doença que se apanha através de marido" (participante africana). "Eu não tenho idéia disso, porque problema grave nunca vi. Eu não conheço e não vi” (participante africana).

As participantes brasileiras, por sua vez, demonstram um nível de conhecimentos sobre DST mais elevado; conseqüentemente, afirmam ser fundamental existir um maior investimento na área da informação sobre os métodos de prevenção: “A dificuldade aqui é que tem poucas explicações (...) sobre as camisinhas, sobre a AIDS. (...) Se fala na AIDS, mas (...) tem outras doenças que também são sexualmente transmissíveis, a candidiase, que é uma coisinha boba, mas que pode agravar, ou mesmo a sífilis. Então, isso não tem sido divulgado" (participante brasileira).

No decorrer de suas intervenções, as participantes brasileiras reconhecem também a importância de realizar o teste de diagnóstico do HIV/ AIDS e comentam que "os médicos até perguntam por que a gente quer fazer tanto exame (...)” (participante brasileira). Por outro lado, algumas salientam, como um exemplo positivo, a facilidade com que é possível realizar o teste do HIV em Portugal: "É muito fácil e mais barato aqui" (participante brasileira). “... Aqui também tem os centros que uma pessoa vai. (...) Você vai, fala que quer fazer o exame, o médico vem e faz o exame de imediato" (participante brasileira).

As diferenças relativas a esta matéria encontradas nos grupos focais com mulheres africanas e brasileiras poderão, em parte, ser explicadas pela diferença no nível educacional entre esses dois grupos, já que tem sido apontado que um maior conhecimento sobre planejamento familiar, contracepção e DST está associado a níveis educacionais mais elevados 37,38,39. Afinal, segundo Norman \& Gebre 40 e Worthington \& Myers 41, os indivíduos que apresentam níveis maiores de conhecimento tendem a perceber a doença como um risco pessoal e a reconhecer a importância de tomar medidas protetoras.

As mulheres africanas, além de demonstrarem desconhecimento em relação a esta temática, argumentam freqüentemente que a sua vulnerabilidade às DST está associada ao comportamento sexual adotado pelos seus parceiros: "É quase impossível [exigir fidelidade]. Basta eles saírem na esquina, encontrar uma menina e já está. E a gente não sabe se eles usam camisa, para evitar vários tipos de doença que há. E eles podem apanhar e transmitir a nós, mesmo nós sendo fiéis a eles" (participante africana).

No mesmo sentido, salientam ainda a dificuldade de adotarem comportamentos preventivos no contexto de uma relação conjugal: "Eu gostaria de fazer uma pergunta: uma dona de casa, que o seu esposo pula a cerca com mulheres diferentes e tem relação com a sua esposa sem preservativo (...). Pronto, corremos um maior risco e, então, que tipo de precaução devemos tomar?" (participante africana).

Nas discussões dos grupos focais, as participantes africanas, principalmente as que têm relações conjugais estáveis, salientam a dificuldade de "negociar" o uso do preservativo com o parceiro: "Nós somos donas de casa, não costumamos usar e, de repente, começamos a dizer 'tens de usar preservativo', eles não aceitam. Temos é de prevenir de outra maneira" (participante africana). "Mas uma dona de casa, que já tem uma vida sexual com o marido, no nosso caso, que tem dois, três, quatro filhos, que nunca usou preservativo, como é que faz?" (participante africana).

Um pequeno grupo de mulheres africanas atribui ao homem a responsabilidade do uso do preservativo: "O homem deve ter a consciência, se sabe que tem outra mulher ou parceira ou namorada” (participante africana). "Eu acho que os homens é que deviam ter essa consciência, já que têm uma mulher em casa mais os seus filhos, e se gosta de pular a cerca, quando está com a outra mulher, tem de usar preservativo. Porque nós, donas de casa, somos inocentes, não sabemos o que eles vão fazer quando vão trabalhar, quando vão passear, nós não sabemos" (participante africana).

É visível que a negociação do preservativo nas relações heterossexuais é freqüentemente difícil. De acordo com alguns autores 42,43,44,45,46, em muitos casos, as mulheres africanas acreditam que têm muito pouco controle sobre o comportamento sexual de seus parceiros e oportunida- 
des limitadas para adotar medidas de prevenção da infecção pelo HIV (como o uso do preservativo). De fato, no que diz respeito à adoção de medidas preventivas, parecem existir diferenças na capacidade da mulher e do homem de se protegerem 47 , pois as normas sócio-culturais colocam, muitas vezes, a mulher em posições mais vulneráveis em relação à proteção das DST 48 . Por este motivo, segundo alguns autores 20,49,50, é essencial considerar a influência das questões de gênero e as diferenças de poder na tomada de decisão sobre o uso de preservativo, na medida em que, muitas vezes, esta é uma decisão unilateral do homem.

O desenvolvimento de programas efetivos de prevenção e promoção do uso do preservativo requer que os comportamentos sexuais e a negociação do "sexo seguro" sejam contextualizados na realidade em que a comunidade está inserida, determinada por sua situação epidemiológica, pela mobilidade, pelos tabus e crenças religiosas, pelas dinâmicas de gênero e poder implícitas nesta problemática e pelos fatores sociais, econômicos e culturais 20,43,51,52. Portanto, é necessário promover uma imagem social do preservativo a qual reafirme a sua eficácia no âmbito da prevenção, bem como a sua adequação em uma relação estável, já que tem sido reconhecido que as intervenções na área da saúde sexual e reprodutiva devem envidar esforços para envolver também os homens neste processo, pois em muitos contextos é o seu comportamento que direta ou indiretamente afeta a saúde das mulheres 53,54.

Por outro lado, uma das participantes refere que as próprias mulheres, por vezes, igualmente adotam comportamentos de risco: "Também há mulheres que têm namorado fora. Há mulheres que têm amante fora. E podem apanhar doenças $e$, às vezes, não sabem" (participante africana).

Ainda que o "uso do preservativo" seja reconhecido como uma estratégia preventiva em face das DST, nem todas as participantes (particularmente as africanas) reconhecem ou confiam em sua eficácia: "Em Cabo Verde, apareceu um miúdo na rua com uma caixa, e a gente disse: 'Balão?'. E isso fê-lo rir tanto. Eu soprei esse balão para enfeitar a casa. Meu primo já estava, ele disse que essa coisa não dá para a gente pôr na casa como balão. O balão tem cor e compra na mercearia. Mas esse tem só uma cor e é para uma coisa, e ele não me explicou o que é" (participante africana). “... Há preservativos que vêm furados” (participante africana). "Não são resistentes" (participante africana).

E mesmo que exista consenso por parte das participantes sobre a necessidade do uso do preservativo, algumas mulheres reconhecem que nem sempre o utilizam: “Nós é que temos de de- fender a nossa saúde. Eles [os parceiros] insisteme depois nós, naquela hora, esquecemo-nos e, 'pombas', fazemos sexo sem preservativo. E depois não são problemas só da gravidez, mas das doenças" (participante africana). "Mesmo a gente sendo casada, tem de ter cuidado, não é? Usar a camisinha é coisa que a gente não faz..." (participante brasileira).

No entanto, verifica-se existir um grupo de participantes que demonstra uma atitude assertiva e parece estar decidida a assumir parte do controle em relação à adoção de estratégias preventivas, criticando as mulheres que valorizam em demasia o poder dos parceiros nas relações heterossexuais: "Às vezes, a insistência de uma pessoa é tanta que, se a gente não der um 'stop', eles acabam por [fazer] e o mal é para nós. Eu, por exemplo, (...) com o meu parceiro, ponho sempre preservativo. Ele, às vezes, insiste, e eu digo 'não'. Tem de ser assim" (participante africana).

\section{Considerações finais}

Os resultados deste estudo visam a contribuir para o desenvolvimento de políticas e estratégias de promoção da saúde sexual e reprodutiva em populações imigrantes. Os dados sugerem que as mulheres imigrantes não podem ser consideradas como um grupo homogêneo no que diz respeito aos conhecimentos, atitudes e comportamentos em saúde sexual e reprodutiva. Sendo assim, é preciso que as intervenções nesta área lidem com as diferenças e as necessidades específicas de cada grupo. Tal estratégia poderá conduzir a intervenções mais adequadas aos desejos, valores e necessidades pessoais, criando maior aceitação por parte das populações imigrantes e, conseqüentemente, aumentando a eficácia das ações implementadas.

Na promoção da saúde sexual e reprodutiva, deve-se ter em conta os papéis tradicionais associados ao gênero, às diferenças de poder nas relações heterossexuais e aos fatores culturais, pois podem facilitar ou limitar a adoção de práticas saudáveis. Torna-se imperioso, por isso, considerar a população masculina no desenvolvimento e na implementação de estratégias.

Os dados obtidos indicam, também, que as decisões nessa área não são tomadas individualmente, mas envolvem um complexo contexto sócio-cultural. Assim, as opções estão muito dependentes dos fatores associados ao processo de migração, incluindo os constrangimentos sócioculturais, econômicos e políticos. O reconhecimento e a compreensão dos complexos determinantes que envolvem a saúde sexual reprodutiva poderão contribuir para a construção de progra- 
mas de promoção mais efetivos. Logo, é necessária uma abordagem holística da saúde sexual e reprodutiva que contemple as várias dimensões do conceito e o bem-estar das populações.

$\mathrm{O}$ isolamento social das populações imigrantes, muitas vezes reflexo das zonas geográficas de residência e da estigmatização e discriminação quanto à sua condição sócio-econômica ou origem étnica, condiciona a sua integração na sociedade de acolhimento. Tal isolamento mantém as populações segregadas em grupos, o que pode contribuir para a manutenção ou agravamento das desigualdades sociais e dos fatores de risco na área da saúde sexual e reprodutiva. Se pretendemos reduzir as iniqüidades em saúde, bem como os fatores e as condições de risco, é necessário intervir nos determinantes sociais, sobretudo com a criação de condições de acolhimento mais positivas.

\section{Resumo}

A saúde sexual e reprodutiva representa uma das principais preocupações da Saúde Pública, pois afeta a saúde e o bem-estar dos indivíduos e compromete o nível social e econômico das sociedades. Muitos problemas relacionados à saúde sexual e reprodutiva são acentuados em grupos socialmente desfavorecidos, como os imigrantes. Esta investigação aprofunda o conhecimento de alguns aspectos sobre a saúde sexual e reprodutiva de imigrantes brasileiras e africanas em Portugal, particularmente em relação ao uso de métodos contraceptivos, infecções sexualmente transmissíveis e uso do preservativo. A coleta de dados se deu a partir da realização de grupos focais com $\mathrm{mu}$ lheres imigrantes, em idade fértil, residentes em Portugal e provenientes do Brasil ou de Países Africanos de Língua Oficial Portuguesa. Os resultados permitem compreender as percepções e conhecimentos das participantes em relação às temáticas abordadas e determinar os aspectos a serem levados em conta para melhor atender suas necessidades de saúde sexual e reprodutiva.

Saúde Sexual e Reprodutiva; Saúde da Mulher; Emigrantes e Imigrantes; Prevenção de Doenças Transmissiveis
As políticas de imigração, portanto, devem promover a inclusão social, reduzir as atitudes discriminatórias e contribuir para a redução dos fatores associados à vulnerabilidade das populações imigrantes no que se refere à saúde sexual e reprodutiva. As populações imigrantes, por sua vez, devem poder beneficiar-se do mesmo tipo de fatores protetores da população em geral, tal como recursos sócio-econômicos, condições habitacionais, proteção social e laboral, além de igualdade de oportunidades na educação e saúde.

Por fim, cabe salientar que as conclusões desta investigação devem ser interpretadas como uma tentativa de "abrir e/ou manter as portas abertas”, já que certamente muitas das questões aqui trazidas constituem-se em aspectos que deverão ser mais e melhor desenvolvidos em trabalhos futuros.

\section{Colaboradores}

C. M. F. Rocha contribuiu na realização do projeto e redação da versão do artigo a ser publicada. S. F. Dias colaborou na concepção e realização do projeto e aprovação final da versão do artigo a ser publicada. A. F. Gama participou na redação do relatório final do projeto e revisão da versão do artigo a ser publicada.

\section{Agradecimentos}

Agradecemos o apoio financeiro recebido pelo Alto Comissariado para a Imigração e Diálogo Intercultural (IP - ACIDI), Portugal. Agradecemos também a Alexandra Maximiano, por sua ativa participação em todas as etapas do projeto, e a Ana Claudia Meira, pela revisão cuidadosa do artigo. Por fim, o nosso profundo agradecimento às mulheres que participaram da pesquisa $e$ que partilharam as suas idéias e experiências de vida. 


\section{Referências}

1. United Nations Population Fund. A passage to hope: women and international migration. http:// www.unfpa.org.br/Arquivos/sowp06-en.pdf (acessado em 06/Ago/2008).

2. Braveman P, Gruskin S. Poverty, equity, human rights and health. Bull World Health Organ 2003; 81:539-45.

3. Jolly S, Reeves H. Gender and migration: overview report. Brighton: Bridge/Institute of Development Studies; 2005.

4. International Organization for Migration. Migration health report. http://www.iom.int/iomweb site/Publication/ServletSearchPublication?event= detail\&id=4494 (acessado em 15/Mar/2007).

5. United Nations. Report on the world social situation 2003. Social vulnerability: sources and challenges. New York: Department of Economic and Social Affairs, United Nations; 2003.

6. United Nations Population Fund. Programme of action of the International Conference on Population and Development. New York: United Nations Population Fund; 1996.

7. World Health Organization. Education and treatment in human sexuality: the training of health professionals. Geneva: World Health Organization; 1975. (Technical Report Series, 572).

8. World Health Organization. Defining sexual health: report of a technical consultation on sexual health. Geneva: World Health Organization; 2006.

9. Janssens K, Bosmans M, Temmerman M. Sexual and reproductive health and rights of refugee women in Europe. Ghent: International Centre for Reproductive Health/World Health Organization/ Ghent University; 2005.

10. Fundo das Nações Unidas para a População. A situação da população mundial 2005. A promessa de igualdade: equidade em matéria de gênero, saúde reprodutiva e objetivos de desenvolvimento do milênio. New York: Fundo das Nações Unidas para a População; 2005.

11. World Health Organization. World health report 2002: reducing risks, promoting healthy life. Geneva: World Health Organization; 2002.

12. World Bank. Engendering development: thought gender equality in rights, resources and voice. New York: Oxford University Press/World Bank; 2001.

13. Rademakers J, Mouthaan I, De Neef M. Diversity in sexual health: problems and dilemmas. Eur J Contracept Reprod Health Care 2005; 10:207-11.

14. Joint United Nations Programme on HIV/AIDS. 2006 Report on the global AIDS epidemic. Geneva: Joint United Nations Programme on HIV/AIDS; 2006.

15. Essén B, Hanson BS, Ostergren PO, Lindquist PG, Gudmundsson S. Increased perinatal mortality among sub-Saharan immigrants in a city-population in Sweden. Acta Obstet Gynecol Scand 2000; 79:737-42.

16. Mosher WD, Martinez GM, Chandra A, Abma JC, Willson SJ. Use of contraception and use of family planning services in the United States: 1982-2002. Adv Data 2004; 350:1-36.
17. Kornosky JL, Peck JD, Sweeney AM, Adelson PL, Schantz SL. Reproductive characteristics of Southeast Asian immigrants before and after migration. J Immigr Minor Health 2007; 10:135-43.

18. Lindstrom D, Hernández C. Internal migration and contraceptive knowledge and use in Guatemala. Int Fam Plan Perspect 2006; 32:146-53.

19. Wolff H, Epiney M, Lourenco A, Costanza M, Delieutraz-Marchand J, Andreoli N, et al. Undocumented migrants lack access to pregnancy care and prevention. BMC Public Health 2008; 8:93-103.

20. Woollett A, Marshall H, Stenner P. Young women's accounts of sexual activity and sexual/reproductive health. J Health Psychol 1998; 3:369-81.

21. Sormati M, Pereira L, El-Bassel N, Witte S, Gilbert L. The role of community consultants in designing an HIV prevention intervention. AIDS Educ Prev 2001; 13:311-28.

22. Serviço de Estrangeiros e Fronteiras. Relatório de actividades 2007 - imigração, fronteiras e asilo. Lisboa: Departamento de Planeamento e Formação, Serviço de Estrangeiros e Fronteiras; 2007.

23. Instituto Nacional de Estatística. XIV recenseamento geral da população, 2001. Lisboa: Instituto Nacional de Estatística; 2001.

24. Diniz E. A mulher brasileira na imigração em Portugal. In: SOS Racismo, editor. Imigração e etnicidade: vivências e trajectórias de mulheres em Portugal. Lisboa: SOS Racismo; 2004. p. 199-203.

25. Peixoto J. Mulheres migrantes: percursos laborais e modos de inserção socioeconómica das imigrantes em Portugal. Lisboa: Instituto Superior de Economia e Gestão; 2006.

26. Gil AC. Métodos e técnicas de pesquisa social. 4ạ Ed. São Paulo: Editora Atlas; 1995.

27. Frith H. Focusing on sex: using focus groups in sex research. Sexualities 2000; 3:275-97.

28. Kitzinger J. Focus groups with users and providers of health care. In: Pope C, Mays N, editors. Qualitative research in health care. 2nd Ed. London: British Medical Association; 2000.

29. Marcell A, Raine T, Eyre S. Where does reproductive health fit into the lives of adolescent males? Perspect Sex Reprod Health 2003; 35:180-6.

30. Lobdell D, Gilboa S, Mendola P, Hesse B. Use of focus groups for the environmental health researcher. J Environ Health 2005; 67:36-42.

31. Peterson-Sweeney K. The use of focus groups in pediatric and adolescent research. J Pediatr Health Care 2005; 19:104-10.

32. Bardin L. Análise de conteúdo. Lisboa: Edições 70; 1988.

33. Tong C, Chen J, Cheng L. Survey on causes of induced abortion in Shanghai. Shanghai Medical Journal 1999; 22:345-53.

34. Zhao D, Zheng Z, Zhang L. Status and needs of sexual health among women migrant workers in Taiyuan. In: Gao E, Lou C, Tu X, Iqbal S, editors. Status, perspective and strategy of reproductive health among adolescents and unmarried youth. Shanghai: The Second Military Medical University Press; 2002. p. 231-43. 
35. London A, Robles A. The co-occurrence of correct and incorrect HIV transmission knowledge and perceived risk for HIV among women of childbearing age in El Salvador. Soc Sci Med 2000; 51: 1267-78.

36. Dias S. Contribuição para o conhecimento dos comportamentos sexuais nos adolescentes: promoção da saúde sexual e prevenção do VIH/SIDA [Tese de Doutorado]. Lisboa: Instituto de Higiene e Medicina Tropical, Universidade Nova de Lisboa; 2005.

37. Wong F, Campsmith M, Nakamura G, Crepaz N, Begley E. HIV testing and awareness of care-related services among a group of HIV-positive Asian Americans and Pacific Islanders in the United States: Findings from a supplemental HIV/AIDS surveillance project. AIDS Educ Prev 2004; 16:440-47.

38 Burns F, Mercer C, Copas A, Johnson A, Morison L, Wellings K, et al. Factors associated with HIV testing among black Africans in Britain. Sex Transm Infect 2005; 81:494-500.

39. Dias S, Matos M, Gonçalves A. AIDS-related stigma and attitudes towards AIDS-infected people among adolescents. AIDS Care 2006; 18:208-14

40. Norman L, Gebre Y. Prevalence and correlates of HIV testing: an analysis of university students in Jamaica. MedGenMed 2005; 7:70.

41. Worthington C, Myers T. Factors underlying anxiety in HIV testing: risk perceptions, stigma, and the patient-provider power dynamic. Qual Health Res 2003; 13:636-55.

42. Weeks R, Schensul J, Williams S, Singer M, Grier M. AIDS prevention for African American and Latina women: Building culturally and gender appropriate intervention. AIDS Educ Prev 1995; 7:251-63.

43. Wingood G, DiClemente R. Application of the theory of gender and power to examine HIV-related exposures, risk factors, and effective interventions for women. Health Educ Behav 2000; 27:539-65.

44. Lauby J, Semaan S, O'Connell A, Person B, Vogel A. Factors related to self-efficacy for use of condoms and birth control among women at risk for HIV infection. Women Health 2001; 4:71-91.
45. Williams S, Gardos P, Ortiz-Torres B, Toss S, Ehrhardt A. Urban women's negotiation strategies for safer sex with their male partners. Women Heatlh 2001; 33:133-48.

46 Dias S, Matos M, Gonçalves A. Conhecimentos, atitudes e comportamentos face ao VIH numa comunidade migrante - implicações para a intervenção. Psicologia, Saúde \& Doença 2002; 3:89-102.

47. Buysse A, Van Oost P. 'Appropriate' male and female safer sexual behavior in heterosexual relationships. AIDS Care 1997; 9:549-61.

48. Wingood G, DiClemente R. The theory of gender and power. In: DiClemente RJ, Crosby RA, Kegler MC, editors. Emerging theories in health promotion practice and research: strategies for improving public health. San Francisco: Jossey-Bass; 2002. p. 313-45.

49. Associação para o Planeamento da Família. Migrações internacionais: género e saúde sexual e reprodutiva. http://www.duplaoportunidade.org/IMG/ pdf/Migracoes.pdf (acessado em 23/Jul/2006).

50. Wall K, Nunes C, Matias A. Immigrant women in Portugal: migration trajectories, main problems and policies. Lisboa: Instituto de Ciências Sociais, Universidade de Lisboa; 2005.

51. Mann J. AIDS in the world: a global epidemic out of control? Cambridge: Harvard School of Public Health; 1992.

52. Kelly J, Murphy D, Sikkema K, Kalichaman S. Psychological interventions to prevent HIV infection are urgently needed: new priorities for behavioral research in the second decade of AIDS. Am Psychol 1993; 48:1023-34.

53. Lawrence J, Elderidge G, Reitman D, Little C, Shelby $\mathrm{M}$, Brasfield T. Factors influencing condom use among African American women: implications for risk reduction interventions. Am J Community Psychol 1998; 26:7-28.

54. Elwy A, Hart G, Hawkes S, Petticrew M. Effectiveness of interventions to prevent STI/HIV in heterosexual men: a systematic review. Arch Intern Med 2002; 162:1818-30.

Recebido em 06/Set/2009

Versão final reapresentada em 01/Fev/2010

Aprovado em 18/Mar/2010 